\title{
Discriminative Effects of Social Skills Training on Facial Emotion Recognition among Children with Attention-Deficit/Hyperactivity Disorder and Autism Spectrum Disorder
}

\author{
Ji-Seon Lee ${ }^{1}, \mathrm{Na}-\mathrm{Ri} \mathrm{Kang}^{2}$, Hui-Jeong $\mathrm{Kim}^{2}$, and Young-Sook Kwak ${ }^{3}$ \\ ${ }^{1}$ Balgeunmaeum Psychiatric Clinic, Gwangju, Korea \\ ${ }^{2}$ Department of Psychiatry, Jeju National University Hospital, Jeju, Korea \\ ${ }^{3}$ Department of Psychiatry, Jeju National University School of Medicine, Jeju, Korea
}

\begin{abstract}
Objectives: This study investigated the effect of social skills training (SST) on facial emotion recognition and discrimination in children with attention-deficit/hyperactivity disorder (ADHD) and autism spectrum disorder (ASD).

Methods: Twenty-three children aged 7 to 10 years participated in our SST. They included 15 children diagnosed with ADHD and 8 with ASD. The participants' parents completed the Korean version of the Child Behavior Checklist (K-CBCL), the ADHD Rating Scale, and Conner's Scale at baseline and post-treatment. The participants completed the Korean Wechsler Intelligence Scale for Children-IV (K-WISC-IV) and the Advanced Test of Attention at baseline and the Penn Emotion Recognition and Discrimination Task at baseline and post-treatment.

Results: No significant changes in facial emotion recognition and discrimination occurred in either group before and after SST. However, when controlling for the processing speed of K-WISC and the social subscale of K-CBCL, the ADHD group showed more improvement in total ( $\mathrm{p}=0.049)$, female ( $\mathrm{p}=0.039)$, sad ( $\mathrm{p}=0.002)$, mild ( $\mathrm{p}=0.015)$, female extreme ( $\mathrm{p}=0.005)$, male mild ( $\mathrm{p}=0.038)$, and Caucasian $(\mathrm{p}=0.004)$ facial expressions than did the ASD group.

Conclusion: SST improved facial expression recognition for children with ADHD more effectively than it did for children with ASD, in whom additional training to help emotion recognition and discrimination is needed.
\end{abstract}

Key Words: Attention-deficit/hyperactivity disorder; Autism spectrum disorder; Social skills training; Facial recognition.

Received: February 27, 2018 / Revision: May 2, 2018 / Accepted: May 31, 2018

Address for correspondence: Young-Sook Kwak, Department of Psychiatry, Jeju National University School of Medicine, 15 Aran $13-$ gil, Jeju 63241 , Korea Tel: +82-64-717-1850, Fax: +82-64-717-1849, E-mail: yskcpy@jejunu.ac.kr

\section{INTRODUCTION}

Social skills are specific behaviors used to establish positive social relationships, encompassing both the verbal and non-verbal behaviors required for effective communication. During childhood, social skills are associated with multiple aspects of development, including relationships with peers, academic achievement, and mental health; therefore, children with deficits in social skills experience various difficulties in development [1].

The ability to recognize the emotions behind facial expressions is essential for understanding the emotions and intentions of others and responding appropriately. This is important for establishing and maintaining positive interpersonal

This is an Open Access article distributed under the terms of the Creative Commons Attribution Non-Commercial License (https://creativecommons.org/licenses/by-nc/4.0) which permits unrestricted non-commercial use, distribution, and reproduction in any medium, provided the original work is properly cited. relationships, including those with peers, and can also act as a criterion for determining which social skills to use and when to use them [2].

Emotion recognition is fundamental to the development of social interactions, and social interactions also help to develop emotion recognition by providing more opportunities to recognize verbal and non-verbal emotional cues [3]. Autism spectrum disorder (ASD) is characterized by difficulties in social interaction and verbal and non-verbal communication; thus, individuals with ASD not only have difficulty recognizing facial expressions, but also fewer opportunities to develop this ability. Several studies have reported that individuals with ASD have difficulty recognizing social cues present in tone and physical posture, and there is also evidence of impaired recognition of facial expressions [3]. Children with ASD tend to have particular difficulty recognizing expressions showing negative emotion. Although this im- 
proves over time, similar difficulty recognizing negative emotions such as fear and sadness has been observed in adults with ASD as well [4].

Social impairment is also observed in individuals with attention-deficit/hyperactivity disorder (ADHD), for which there are two broad explanations. Some studies report that this impairment is due to missed opportunities for social interaction as well as negative evaluation by others as a result of impulsivity and lack of attention; meanwhile, other studies have reported that individuals with ADHD have decreased ability to properly interpret and respond to their own and others' emotions [5]. Past studies have provided evidence that, compared to typically developing children, children with ADHD have impaired abilities to recognize negative emotions that can signal disapproval or threat, such as anger, fear, or sadness. As a result of this impairment, children with ADHD cannot stop engaging in socially unaccepted behaviors and experience continual difficulties responding to negative feedback and maintaining positive relationships.

Although this suggests that the two conditions share difficulties in emotion recognition, some studies have reported that individuals with ADHD showed better performance in a facial expression recognition task than individuals with ASD [6], whereas other studies have reported that although there was a difference in facial expression recognition between individuals with ASD and typically developing individuals, there was no significant difference between individuals with ASD and ADHD [7]. This discrepancy has still not been clearly resolved. Domestically, Lee et al. [8] studied the difference in recognition of facial expressions and discrimination of expression intensity between children with $\mathrm{ADHD}$ and ASD and reported that the ADHD group was better than the ASD group at recognizing happy and sad expressions, female facial expressions, and strong expressions, but showed similar difficulties to the ASD group in recognizing male facial expressions and gentle expressions. There was no significant difference between the groups for discrimination of expression intensity. This indicates that children with ADHD are better than those with ASD at recognizing and discriminating large differences of expression but show similar difficulties in recognizing and discriminating smaller differences. Since there are no other domestic studies directly comparing emotion recognition between ASD and ADHD, further research is needed.

Social skills can be learned through educational programs, which have been demonstrated to be effective. Social skills training (SST) has been implemented since its development in the 1970s, in which children practice learned verbal and non-verbal techniques in groups. Although there was initially some debate about the efficacy of SST in children with
ADHD, relatively recent studies have reported that SST helps to improve sociability in children with ADHD [9]. Domestically, Paek et al. [9] applied an SST program to 9 children and observed improvements in social skills and self-esteem of the children with ADHD; Hwang and Kwak [10] implemented SST in children with ADHD and ASD aged 6-12 visiting as outpatients, and found that the intervention was effective for the ADHD group in all areas, including not only social skills but also problems with attention, internalization, and externalization; whereas for the ASD group, significant improvements were primarily seen in social skills. For children with ASD, SST is useful for improving social ability and learning social skills. It has been implemented using various techniques, and with the accumulation of a large amount of empirical evidence, it is currently recognized as an evidence-based practice [11]. However, in Korea, there have not yet been any studies using structured neurocognitive tests to quantitatively analyze whether facial expression recognition ability improves after SST in children with ADHD and ASD.

To this end, we designed our study with the following hypotheses. First, we expected that both disease groups would show positive changes in sociability-related measures after SST. Second, we predicted that the ADHD group would show greater changes in sociability-related measures than the ASD group after SST. Third, we predicted that facial expression recognition and discrimination of expression intensity would improve in both groups after SST. Finally, we hypothesized that the ADHD group would show a greater improvement in facial expression recognition than the ASD group after SST, but that there would be little or no difference between the groups in improvement in discrimination of expression intensity.

In order to test these hypotheses, we retrospectively analyzed school-age children with ADHD or ASD before and after SST, examining changes in affective behavior, using structured neurocognitive tests to evaluate changes in facial expression recognition and discrimination of expression intensity, and investigating any differences in the extent of these changes between the two groups.

\section{METHODS}

\section{Participants}

The subjects in this study consisted of children aged 7-10 years who had visited the Jeju National University Hospital Department of Child and Adolescent Psychiatry as outpatients, had been diagnosed with ADHD or ASD by the attending child and adolescent psychiatrist, and had participated in the hospital's SST program from July 2013 to February 
2017. Of these, there were 8 children diagnosed with ASD and 15 children diagnosed with ADHD. The children diagnosed with ASD satisfied the diagnosis criteria of the Diagnostic and Statistical Manual of Mental Disorders-Fifth Edition (DSM-5), and the children diagnosed with ADHD satisfied the diagnostic criteria of both the DSM- 5 and the KiddieSchedule for Affective Disorders and Schizophrenia-Present and Lifetime Version (K-SADS-PL). In order to minimize the effects of medication on the outcome, we excluded children who underwent a change in their dose or type of medication during the SST period. We also excluded children who had participated in less than 12 of the total 24 sessions or who had an overall intelligence score of less than 70 . This study was approved by the Institutional Review Board at Jeju National University Hospital (IRB No. JEJUNUH 2016-05-008).

\section{Methods}

SST was composed of separate child and parent groups, led by a team of one speech and language therapist and one child and adolescent psychiatrist. The child groups convened for a total of 24 sessions for 1.5 hours per session, with 1 session per week. The parent groups convened for a total of 12 sessions for 40 minutes per session, 1 session every two weeks. The child groups were divided into younger groups (i.e., 1 st-3rd grade) and older groups (i.e., 4 th-6th grade). There were approximately 10 children in each group, never exceeding 12 children.

Before beginning SST, children were examined using the Korean-Wechsler Intelligence Scale for Children-IV (K-WISCIV), the Advanced Test of Attention (ATA), the Penn Emotion Recognition Task (ER40), and the Penn Emotion Discrimination Task (EDF40). The children's primary caregivers were examined using the Korean-Child Behavior Checklist (K-CBCL), the ADHD Rating Scale (ARS), the Abbreviated Conner's Rating Scale (CRS), Matson Evaluation of Social Skills with Youngsters (MESSY), and the Social Skills Rating System (SSRS). All the assessments except the K-WISC-IV were repeated immediately after the end of the last SST session.

\section{Social skills training (SST) program}

The SST program in this study was based on the program devised by Pfiffner and McBurnett for use in children aged 6-11 years old [12].

Our SST program consisted of 12 topics, with each topic lasting 1-2 weeks. In the child sessions, the first 10-15 minutes was used for a review of the last week's tasks and a discussion of things that had happened in the last week; the main part of the session consisted of debate, role-play, and play activities relating to the current week's topic; and the session ended with handing out new tasks as well as rewards
Table 1. Overview of social skills training

\begin{tabular}{|c|c|}
\hline Session & Topics \\
\hline \multirow[t]{3}{*}{1} & Program overview \\
\hline & Making group rules \\
\hline & Presenting rewards \\
\hline \multirow[t]{3}{*}{2} & Socializing to make friends \\
\hline & Social entry skills- good and bad friendship \\
\hline & characteristics \\
\hline $3-4$ & Having fun with a friend \\
\hline $5-6$ & Having a positive attitude regarding the result \\
\hline \multirow[t]{2}{*}{$7-8$} & Participating in conversations \\
\hline & Becoming a friend people want to speak to \\
\hline \multirow[t]{3}{*}{$9-10$} & Recognizing emotions in yourself and others \\
\hline & Knowing the difference between positive and \\
\hline & negative emotions \\
\hline $11-12$ & Dealing with negative emotions \\
\hline $13-14$ & Praising friends \\
\hline $15-16$ & Know your strengths and weaknesses \\
\hline $17-18$ & Learning problem solving skills \\
\hline $19-20$ & Dealing with teasing \\
\hline $21-22$ & Learning to be self-assertive \\
\hline 23 & Apologizing to friends \\
\hline 24 & Review and party \\
\hline
\end{tabular}

for performances during the day's session or during previous tasks. Table 1 shows the detailed content of the SST program used in this study.

The aim of the parent groups was to help the children generalize the social skills learned during SST at home and school. At the start of each session, parents were each given a report of their child's observed behavior during the last session, and they shared their opinions regarding their child's behavior and task performance at school and at home since the last session. Next, the parents were provided with information about what social skills their child would learn that week and what the parents could do to help them, and they were also taught methods to handle and correct problematic behaviors.

\section{Instruments}

Kiddie-Schedule for Affective Disorders and SchizophreniaPresent and Lifetime Version (K-SADS-PL)

This is a semi-structured interview developed to evaluate the current and lifetime morbidity of psychiatric diseases and the severity of symptoms in children and adolescents, based on DSM-IV diagnostic criteria. The original instrument was developed by Kaufman et al. [13], and the Korean version was adapted by Kim et al. [14] and has been shown to be reliable and valid for ADHD, tic disorders, oppositional defi- 
ant disorder, depression, and anxiety disorder. In relevant studies, the K-SADS-PL showed good to excellent validity and fair to excellent reliability for ADHD diagnoses, meaning that it has been shown to be effective for diagnosing ADHD.

\section{Korean-Wechsler Intelligence Scale for Children-IV (K-WISC-IV)}

The WISC-IV, developed by Wechsler, was adapted for the circumstances in Korea by the Korean Educational Development Institute [15] and is used to evaluate the intelligence of children aged 6 to 16 years and 11 months. The instrument is composed of 4 indices: the Verbal Comprehension Index, the Perceptual Reasoning Index, the Working Memory Index, and the Processing Speed Index. In our study, we used the T-scores for overall intelligence and the individual indices and restricted our study to children with an overall intelligence score of 70 or higher.

\section{Korean-Child Behavior Checklist (K-CBCL)}

The CBCL was developed by Achenbach in 1991 as an instrument used to evaluate the overall affect, behavioral problems, and social adjustment of children. Domestically, Oh and Lee [16] prepared a Korean adaptation and analyzed the reliability and validity. The instrument is composed of 13 scales for problematic behavior symptoms, including being withdrawn/depressed, somatic complaints, being anxious/ depressed, social problems, thought problems, attention problems, rule-breaking behavior, aggressive behavior, internalizing problems, externalizing problems, total problems, sexual problems, and emotional instability, and 3 scales for social competence, including social, school, and total competence. A total of 113 questions were evaluated on 3-point scales. There are parent and teacher versions available, and we used the parent version in our study. The K-CBCL is reported to have an internal consistency of $0.62-0.86$, and the problematic behavior syndrome scales are reported to have a mean test-retest reliability of 0.68 .

\section{ADHD Rating Scale (ARS)}

This instrument was devised by DuPaul [17] in 1991 to evaluate ADHD symptoms in children based on DSM-IV diagnostic criteria. So et al. [18] translated and adapted it into a Korean version and tested its reliability and validity, reporting a reliability of 0.94 for the parent version and 0.96 for the teacher version. Of the 18 total questions, 9 questions relate to symptoms of inattention, while the other 9 questions relate to symptoms of hyperactivity and impulsivity. The parent or teacher evaluates the child's symptoms using a 4 -point scale from 'Rarely or never' (0 points) to 'Always or very often' (3 points).

\section{Abbreviated Conner's Rating Scale (CRS)}

This instrument was developed by Keith Conner's in 1969 to evaluate ADHD symptoms in children. Since then, several modified versions have been produced and are widely used in research and clinical settings. The Abbreviated CRS consists of 10 total questions evaluated on a 4-point scale from $0-3$ points and is used especially often. Oh and Lee [19] translated and standardized the instrument in order to produce a Korean version, and reported cutoff scores of 17 points for the teacher version and 16 points for the parent version.

\section{Advanced Test of Attention (ATA)}

The ATA was developed and standardized by Shin et al. [20] in order to objectively evaluate diagnosis and treatment effects for individuals with ADHD. The instrument can be used for children and adolescents aged 5-15 years in order to examine both visual and auditory attention. Omission errors, commission errors, mean of reaction time, and standard deviation of reaction time (response time variability) are measured. Omission errors are used to evaluate sustained attention, commission errors are used to evaluate sustained attention and impulsivity, and mean reaction time and response time variability are used to evaluate information processing speed and response inconsistency. T-scores were calculated for the 4 variables, and ADHD was suspected to be present when there was at least one variable with a T-score over 70 points. In a discriminant analysis by Shin et al. [20] the ATA discriminated between typically developing children and children with ADHD with $96.7 \%$ accuracy, demonstrating that this is a reliable and valid instrument for assessing ADHD.

\section{Matson Evaluation of Social Skills with Youngsters (MESSY)}

MESSY was developed by Matson et al. in 1983 [21] in order to measure social skills in children, and was adapted into a Korean version by Park and Oh [22]. A teacher or parent evaluates a total of 64 questions on a scale from $1-5$. The questions, which are designed to evaluate the extent of the child's social strengths and problems, were scored by the child's parent in our study. Higher scores are associated with greater proficiency in social relationships. The reliability of this instrument has been reported to be 0.93 [23].

\section{Social Skills Rating System (SSRS)}

This instrument was developed by Gresham and Elliott in 1990 [24] in order to measure social skills in children aged 3-18 years. Either the child, a parent, or a teacher grades each 
question on a scale from $0-2$. There are three scales (social skills scale, problem behaviors scale, academic competence scale), but in this study, we only used the 38 questions corresponding to the social skills scale in the parent version. The parent-graded social skills scale is composed of 4 subscales: cooperation, self-assertion, self-control, and responsibility. In domestic research, the reliability of the parent questionnaire was reported to be 0.82 [25].

\section{Penn Emotion Recognition Task (ER40)}

This test was developed by Gur et al. [26] and is included in the Penn Web-Based Computerized Neurocognitive Battery (Web CNP). In the present study, the test was used with the approval of the original author and was conducted by a clinical psychology trainee who had been trained in the test method.

During the test, the child is presented with colored photographs of faces, one at a time, on a computer screen and selects the emotion displayed by the face. The child chooses one of five options (happiness, sadness, anger, fear, and neutral) for a total of 40 photographs. There is no time limit, but the child's choices and response time are recorded for each photograph.

\section{Penn Emotion Discrimination Task (EDF40)}

This test was developed by Erwin et al. [27] and is included in the Web CNP. Rojahn et al. [28] investigated its reliability and validity. In this study, the test was used with the approval of the original author and was conducted by a clinical psychology trainee who had been trained in the test method.

The test method was as follows: the child is presented with a total of 40 pairs of black-and-white photographs of faces, one pair at a time, on a computer screen. The photographs show Caucasian actors expressing emotions (e.g., sadness, happiness, and neutral); each pair shows the same actor expressing the same emotion, and the child has to select the photograph that shows more intense emotion or indicate that the emotion intensity is the same. There is no time limit. Of the 40 pairs of photographs, 4 pairs show the same emotion intensity, and 36 pairs show different emotion intensity; of these, 18 pairs show a happy expression, and 18 pairs show a sad expression.

\section{Data analysis}

In this study, we investigated whether there was any improvement in the ability to recognize types of facial expression and to discriminate expression intensity between the same type of facial expression following SST. In addition, we aimed to determine whether there was any difference in the extent of these changes between the ADHD group and the ASD group. In order to test whether there were any changes in facial expression recognition and discrimination of expression intensity following SST, we used the Wilcoxon signed rank test for each group. To control for variables that could affect expression recognition and discrimination of expression intensity, we used the Mann-Whitney test to analyze the homogeneity of the ADHD and ASD groups for social adjustment, problem behavior, intelligence, and attention. In order to determine whether there were any statistically significant differences between the two groups for the extent of change in expression recognition and expression intensity discrimination, we performed an analysis of covariance, using the Processing Speed Index from the K-WISC-IV and the social competence subscale from the K-CBCL as covariates, since these indices showed clear differences between the two groups in the homogeneity test $(p<0.01)$ and were also expected to be directly related to expression recognition. We used SPSS (version 18; SPSS Inc., Chicago, IL, USA) for all statistical analysis, with a statistical significance level of $\mathrm{p}<0.05$.

\section{RESULTS}

Differences between the ADHD and ASD groups in demographic characteristics, neuropsychological tests, clinical characteristics, and the extent of changes after SST (Table 2 and 3)

Of the 23 children who participated in this study, there were 15 children in the ADHD group (age $8.40 \pm 0.91$ years, 12 males) and 8 children in the ASD group (age $8.0 \pm 0.93$ years, 7 males). There were no statistically significant differences between the two groups in age $(p=0.428)$ or sex $(p=0.565)$.

Although there was no significant difference between the two groups for overall intelligence $(\mathrm{p}=0.101)$, for the K-WISCIV Processing Speed Index, the ADHD group was significantly faster $(p=0.001)$ than the ASD group. For the ATA, the ADHD group also showed a significantly lower auditory response time variability than did the ASD group $(p=0.038)$, but there were no differences in the other subscales.

When we compared clinical characteristics between the two groups, before SST, the ADHD group showed significantly higher CRS scores than the ASD group $(\mathrm{p}=0.040)$ and, though it was not statistically significant, showed a trend for higher ARS scores (mean score ADHD=25.33, ASD=19.63, p=0.056). The ADHD group also showed significantly higher scores than the ASD group for the social subscale of the K-CBCL $(\mathrm{p}=0.005)$.

Following SST, the ADHD group showed positive changes compared to pre-intervention scores on several subscales 
of the K-CBCL: school ( $\mathrm{p}=0.035)$, total competence $(\mathrm{p}=0.009)$, somatic complaints $(\mathrm{p}=0.007)$, social problems $(\mathrm{p}=0.002)$, thought problems $(\mathrm{p}=0.006)$, attention problems $(\mathrm{p}=0.038)$, aggressive behavior $(\mathrm{p}=0.003)$, externalizing behavior $(\mathrm{p}=$ 0.001 ), and total problems ( $\mathrm{p}=0.004)$. The ADHD group also showed improved results for the CRS $(p=0.002)$, ARS $(p=0.001)$,

Table 2. Demographic information and neuropsychological test of children with ADHD and ASD

\begin{tabular}{|c|c|c|c|c|}
\hline & $\operatorname{ADHD}(n=15)$ & $\operatorname{ASD}(n=8)$ & \multirow{2}{*}{ U } & \multirow{2}{*}{$\mathrm{P}$} \\
\hline & Mean (SD) & Mean (SD) & & \\
\hline Age, years & $8.40(0.91)$ & $8.00(0.93)$ & 47.000 & 0.428 \\
\hline Gender (boys), n (\%) & $12(80.0)$ & $7(87.5)$ & $0.204^{\ddagger}$ & $0.565^{\ddagger}$ \\
\hline \multicolumn{5}{|l|}{ WISC-IV } \\
\hline $\mathrm{VCl}$ & $90.53(14.90)$ & $93.50(24.00)$ & 58.500 & 0.925 \\
\hline PRI & $96.13(15.45)$ & 95.25 (20.70) & 51.000 & 0.591 \\
\hline WMI & $85.87(12.46)$ & $79.50(18.33)$ & 34.500 & 0.101 \\
\hline PSI & $88.67(13.73)$ & $70.00(9.30)$ & 13.000 & $0.001^{\dagger}$ \\
\hline$F S I Q$ & $88.07(12.50)$ & 82.38 (20.98) & 34.500 & 0.101 \\
\hline \multicolumn{5}{|l|}{ ATA } \\
\hline Omission errors, visual & $60.43(14.18)$ & $70.71(21.65)$ & 36.500 & 0.360 \\
\hline Commission errors, visual & $69.64(19.59)$ & $79.43(18.12)$ & 33.500 & 0.255 \\
\hline RT, visual & $54.36(11.43)$ & $56.14(9.08)$ & 43.000 & 0.689 \\
\hline RT variability, visual & $61.29(18.38)$ & $62.43(11.91)$ & 39.500 & 0.488 \\
\hline Omission errors, auditory & $58.79(19.54)$ & $72.29(18.79)$ & 25.000 & 0.079 \\
\hline Commission errors, auditory & $67.43(22.76)$ & $81.00(18.55)$ & 32.000 & 0.224 \\
\hline RT, auditory & $44.93(10.71)$ & $35.00(17.86)$ & 32.500 & 0.224 \\
\hline RT variability, auditory & $45.64(10.04)$ & $57.57(11.87)$ & 21.500 & $0.038^{*}$ \\
\hline
\end{tabular}

Data are number or mean (SD) values. ${ }^{*} p<0.05,{ }^{\dagger} p<0.01,{ }^{\ddagger} \chi^{2}$. ADHD: attention-deficit/hyperactivity disorder, ASD: autism spectrum disorder, ATA: Advanced Test of Attention, FSIQ; Full Scale Intelligence Quotient, PRI: Perceptual Reasoning Index, PSI: Processing Speed Index, RT: response time, SD: standard deviation, VCl: Verbal Comprehension Index, WISC-IV: Wechsler Intelligence Scale for Children-IV, WMI: Working Memory Index

Table 3. Group difference of mean score change of clinical characteristics after social skills training between ADHD and ASD groups

\begin{tabular}{|c|c|c|c|c|c|c|}
\hline & \multicolumn{2}{|c|}{ ADHD $(n=15)$, Mean $(S D)$} & \multicolumn{2}{|c|}{$\operatorname{ASD}(n=8)$, Mean $(S D)$} & \multirow{2}{*}{$\mathrm{F}$} & \multirow{2}{*}{$p$} \\
\hline & Pre & Post & Pre & Post & & \\
\hline \multicolumn{7}{|l|}{$\mathrm{K}-\mathrm{CBCL}$} \\
\hline Social & $42.53(8.72)$ & $47.80(10.08)$ & $33.75(3.69)$ & $35.38(10.62)$ & 1.167 & 0.293 \\
\hline School & $46.73(12.04)$ & $54.47(10.85)$ & $46.25(7.07)$ & $45.75(6.50)$ & 2.523 & 0.130 \\
\hline Total social competence & $41.80(9.26)$ & $49.73(9.88)$ & $36.13(4.79)$ & $36.75(11.49)$ & 3.334 & 0.084 \\
\hline Withdrawn & $62.07(7.96)$ & $60.67(15.43)$ & $60.88(7.57)$ & $57.50(7.58)$ & 0.005 & 0.945 \\
\hline Anxious/depressed & $57.60(9.05)$ & $53.33(9.71)$ & $54.50(10.47)$ & $55.63(6.80)$ & 0.327 & 0.574 \\
\hline Somatic complaints & $60.60(9.64)$ & $51.27(8.05)$ & $61.38(7.54)$ & $48.13(9.09)$ & 0.573 & 0.459 \\
\hline Social problems & $64.27(8.60)$ & $52.60(10.71)$ & $65.88(13.81)$ & $65.13(10.60)$ & 4.552 & $0.047^{*}$ \\
\hline Thought problems & $62.93(5.69)$ & $54.93(8.51)$ & $61.63(7.71)$ & $55.88(7.24)$ & 0.642 & 0.433 \\
\hline Attention problems & $63.40(7.94)$ & $56.93(11.30)$ & $66.63(12.52)$ & $60.13(9.63)$ & 0.012 & 0.913 \\
\hline Delinquent behavior & $59.87(9.87)$ & $52.20(11.53)$ & $60.63(9.65)$ & $51.50(11.39)$ & 0.360 & 0.556 \\
\hline Aggressive behavior & $63.87(8.49)$ & $54.47(8.64)$ & $61.75(6.76)$ & $49.88(11.78)$ & 1.646 & 0.216 \\
\hline Internalizing behavior & $62.00(9.37)$ & $57.13(9.59)$ & $61.63(6.35)$ & $55.50(9.81)$ & 0.112 & 0.742 \\
\hline Externalized behavior & $65.13(9.36)$ & $49.47(6.39)$ & $63.00(8.18)$ & $52.88(8.56)$ & 0.135 & 0.717 \\
\hline Total behavior problems & $64.33(9.24)$ & $53.13(8.49)$ & $64.38(8.23)$ & $53.38(12.60)$ & 0.831 & 0.374 \\
\hline ARS & $25.33(7.67)$ & $15.27(8.00)$ & $19.63(14.90)$ & $14.13(11.81)$ & 0.087 & 0.771 \\
\hline CRS & $14.40(5.01)$ & $8.40(4.66)$ & $9.50(9.12)$ & $7.63(5.93)$ & 0.928 & 0.348 \\
\hline MESSY & $112.40(23.68)$ & $120.07(25.51)$ & $107.13(30.33)$ & $100.63(24.68)$ & 4.781 & $0.042^{*}$ \\
\hline SSRS & $31.67(9.01)$ & $40.67(11.11)$ & $27.63(4.00)$ & $36.75(9.91)$ & 0.289 & 0.598 \\
\hline
\end{tabular}

Data are number or mean (SD) values. ${ }^{*} \mathrm{p}<0.05$. ADHD: attention-deficit/hyperactivity disorder, ARS: ADHD Rating Scale, ASD: autism spectrum disorder, CRS: Conner's Rating Scale, K-CBCL: Korean-Child Behavior Checklist, MESSY: Matson Evaluation of Social Skills of Youngsters, SD: standard deniation, SSRS: Social Skills Rating System 
MESSY ( $\mathrm{p}=0.041)$, and SSRS ( $\mathrm{p}=0.007)$. Conversely, after SST, the ASD group showed significant improvements in somatic complaints $(\mathrm{p}=0.036)$ and attention problems $(\mathrm{p}=0.049)$ from the K-CBCL and improvements in the ARS ( $\mathrm{p}=0.018$ ), but there were no significant changes in scales directly related to sociability, which is the main objective of SST.

In order to determine whether there were any significant differences between the two groups with regard to the extent of changes after SST, we controlled for Processing Speed Index from the K-WISC-IV and the social competence subscale from the K-CBCL, since these scales showed clear differences between the two groups before SST $(\mathrm{p}<0.01)$ and were expected to be directly related to facial expression recognition. Auditory response time variability from the ATA $(p=$ $0.038)$ and CRS $(p=0.040)$ were not included in the control variables, since they showed a relatively less clear difference between the two groups and were considered to be unlikely to have a direct effect on facial expression recognition. Even after controlling for the two variables above, the positive changes in the ADHD group after SST were significantly greater than those for the ASD group for the social problems scale from the K-CBCL $(p=0.047)$ and in MESSY $(p=0.042)$.

\section{Differences between the ADHD and ASD groups in improvements in ability to recognize emotions in facial expressions after SST (Table 4)}

Before SST, there was no significant difference between the ADHD and ASD groups in the ability to recognize emotions in facial expressions. After SST, neither group showed significant changes in emotion recognition. However, when we controlled for the Processing Speed Index from the KWISC-IV and the social competence subscale from the KCBCL and compared the change in emotion recognition after SST between the two groups, the ADHD group showed a significantly greater improvement than the ASD group for overall number of correct responses ( $\mathrm{p}=0.049)$, and for number of correct responses for female facial expressions $(p=0.039)$, sad expressions $(p=0.002)$, mild expressions $(p=0.015)$, extreme female expressions ( $\mathrm{p}=0.005)$, mild male expressions $(\mathrm{p}=0.038)$, and Caucasian facial expressions $(\mathrm{p}=0.004)$.

\section{Response time for emotion recognition in ADHD and ASD groups (Table 5)}

When we compared the response time for correctly recognizing emotions in facial expressions, there was no significant difference between the ADHD and ASD groups before SST. After SST, the ADHD group showed significant decreases in overall response time for correct responses $(\mathrm{p}=0.036)$ and in the response time for correct responses for male emotions $(p=0.031)$, neutral emotions $(p=0.023)$, extreme emotions $(\mathrm{p}=0.031)$, and extreme male emotions $(\mathrm{p}=0.012)$. On the other hand, the ASD group showed no significant changes in response times after SST.

When the changes after SST were compared between the two groups while controlling for the Processing Speed Index from the K-WISC-IV and social competence subscale from the K-CBCL, the ADHD group showed a significantly greater decrease than the ASD group for only response time for mild female emotions $(\mathrm{p}=0.043)$.

Table 4. Group difference of mean score change of Penn Emotion Recognition Task (ER40) between ADHD and ASD groups

\begin{tabular}{|c|c|c|c|c|c|c|}
\hline & \multicolumn{2}{|c|}{ ADHD $(n=15)$, Mean (SD) } & \multicolumn{2}{|c|}{ ASD $(n=8)$, Mean (SD) } & \multirow{2}{*}{$\mathrm{F}$} & \multirow{2}{*}{$\mathrm{p}$} \\
\hline & Pre & Post & Pre & Post & & \\
\hline Total & $30.27(2.19)$ & $31.20(3.21)$ & $31.20(3.21)$ & $28.00(2.73)$ & 4.470 & $0.049 *$ \\
\hline Female & $15.53(1.81)$ & $15.80(1.52)$ & $14.13(2.75)$ & $14,25(1.04)$ & 4.976 & $0.039 *$ \\
\hline Male & $14.73(1.22)$ & $15.40(2.67)$ & $13.25(2.82)$ & $13.75(2.55)$ & 1.586 & 0.224 \\
\hline Angry & $4.80(1.37)$ & $4.93(1.22)$ & $4.50(1.85)$ & $5.00(1.41)$ & 0.323 & 0.577 \\
\hline Fear & $5.87(1.25)$ & $5.80(1.47)$ & $5.63(1.92)$ & $5.00(2.51)$ & 0.516 & 0.482 \\
\hline Happy & $7.33(0.62)$ & $7.60(0.63)$ & $6.63(0.92)$ & $7.25(1.04)$ & 1.359 & 0.259 \\
\hline No emotion & $6.87(1.13)$ & $7.27(1.33)$ & $6.25(2.71)$ & $7.00(1.41)$ & 0.000 & 0.985 \\
\hline Sad & $5.40(1.12)$ & $5.60(1.18)$ & $4.38(2.07)$ & 3.75 (1.98) & 13.675 & $0.002^{\dagger}$ \\
\hline Mild & $9.73(1.03)$ & $10.27(1.62)$ & $8.63(1.60)$ & $8.38(1.19)$ & 7.193 & $0.015^{*}$ \\
\hline Extreme & $13.67(1.11)$ & $13.67(1.11)$ & $12.50(2.27)$ & $12.63(1.85)$ & 3.161 & 0.092 \\
\hline Female mild & $5.40(1.18)$ & $5.27(1.10)$ & $5.25(0.89)$ & $4.88(1.25)$ & 0.218 & 0.646 \\
\hline Female extreme & $6.60(0.91)$ & $6.80(0.68)$ & $5.88(1.73)$ & $5.88(1.36)$ & 10.449 & $0.005^{\dagger}$ \\
\hline Male mild & $4.33(0.90)$ & $5.00(1.46)$ & $3.38(1.30)$ & $3.50(1.60)$ & 5.026 & $0.038^{*}$ \\
\hline Male extreme & 7.07 (0.70) & $6.87(0.99)$ & $6.63(1.30)$ & $6.75(1.04)$ & 0.000 & 0.985 \\
\hline Caucasian & $15.33(1.88)$ & $16.40(2.29)$ & $13.63(2.97)$ & $14.13(1.55)$ & 10.932 & $0.004^{\dagger}$ \\
\hline Noncaucasian & 14.93 (1.39) & $14.80(1.52)$ & $13.75(2.43)$ & $13.88(1.96)$ & 0.187 & 0.671 \\
\hline
\end{tabular}

${ }^{*} p<0.05,{ }^{\dagger} p<0.01$. ADHD: attention-deficit/hyperactivity disorder, ASD: autism spectrum disorder, SD: standard deviation 
Table 5. Group difference of mean score change of Penn Emotion Recognition Task (ER40) response time between ADHD and ASD groups

\begin{tabular}{|c|c|c|c|c|c|c|}
\hline \multirow{2}{*}{ CRT } & \multicolumn{2}{|c|}{ ADHD, Mean (SD) } & \multicolumn{2}{|c|}{ ASD, Mean (SD) } & \multirow{2}{*}{$\mathrm{F}$} & \multirow{2}{*}{$\mathrm{p}$} \\
\hline & Pre & Post & Pre & Post & & \\
\hline Total & $2580.50(533.73)$ & $2284.07(497.23)$ & $2382.44(563.18)$ & $2243.81(509.90)$ & 0.422 & 0.524 \\
\hline Female & $2626.53(808.71)$ & $2437.97(611.78)$ & $2413.94(479.54)$ & $2224.19(455.43)$ & 0.478 & 0.498 \\
\hline Male & $2790.10(753.08)$ & $2253.37(620.64)$ & $2373.63(691.27)$ & $2376.69(801.72)$ & 0.040 & 0.843 \\
\hline Angry & $3219.80(1504.74)$ & $2571.77(566.90)$ & $2356.00(494.12)$ & $2494.25(421.61)$ & 1.147 & 0.298 \\
\hline Fear & $3063.17(1213.80)$ & $3120.53(1513.97)$ & $3104.75(1265.16)$ & $2691.19(579.27)$ & 3.898 & 0.064 \\
\hline Happy & $2409.23(544.02)$ & $2141.03(434.33)$ & $2124.56(511.13)$ & 2230.75 (1 116.49$)$ & 0.503 & 0.487 \\
\hline No emotion & $3244.17(1841.53)$ & $2158.67(504.06)$ & $2148.86(679.02)$ & $2312.64(1174.79)$ & 0.052 & 0.823 \\
\hline Sad & $2663.37(793.36)$ & $2535.40(592.57)$ & $3503.06(1716.12)$ & $2792.88(1083.01)$ & 0.034 & 0.856 \\
\hline Mild emotion & $3143.97(1188.80)$ & $2679.07(822.31)$ & $2557.56(762.22)$ & 2519.19 (471.92) & 1.045 & 0.320 \\
\hline Extreme emotion & $2527.07(514.35)$ & $2244.93(495.47)$ & $2355.38(674.85)$ & $2121.00(398.42)$ & 2.122 & 0.162 \\
\hline Female mild & $3174.57(1691.64)$ & $2765.43(787.20)$ & $2613.44(676.53)$ & $2399.56(601.13)$ & 4.728 & $0.043 *$ \\
\hline Female extreme & $2555.17(565.57)$ & $2503.67(926.08)$ & $2636.13(814.02)$ & $2151.06(869.98)$ & 1.955 & 0.179 \\
\hline Male mild & $3213.80(1456.51)$ & $2677.17(1207.33)$ & $2773.06(1246.85)$ & $3429.69(1389.38)$ & 0.946 & 0.344 \\
\hline Male extreme & $2555.70(581.61)$ & $2215.80(568.28)$ & $2315.25(700.43)$ & $2176.63(486.55)$ & 0.000 & 0.995 \\
\hline
\end{tabular}

${ }^{*} \mathrm{p}<0.05$. ADHD: attention-deficit/hyperactivity disorder, ASD: autism spectrum disorder, CRT: correct responses median response time, SD: standard deviation

Table 6. Group difference of mean score change of Penn Emotion Discrimination Task (EDF40) between ADHD and ASD groups

\begin{tabular}{|c|c|c|c|c|c|c|}
\hline & \multicolumn{2}{|c|}{$\operatorname{ADHD}(n=15)$, Mean $(S D)$} & \multicolumn{2}{|c|}{$\operatorname{ASD}(n=8)$, Mean (SD) } & \multirow{2}{*}{$\mathrm{F}$} & \multirow{2}{*}{$\mathrm{p}$} \\
\hline & Pre & Post & Pre & Post & & \\
\hline HAP_CR & $6.53(3.29)$ & $6.40(4.17)$ & $4.00(2.62)$ & $6.50(3.42)$ & 3.458 & 0.079 \\
\hline SAD_CR & $8.80(3.88)$ & $9.20(3.93)$ & $7.00(3.55)$ & $8.00(2.83)$ & 2.315 & 0.145 \\
\hline HAPRTCR, msec & $3401.80(1126.40)$ & $2870.63(952.12)$ & $3218.94(1440.36)$ & $2683.31(1076.25)$ & 0.014 & 0.908 \\
\hline SADRTCR, msec & $2578.57(727.31)$ & $2402.23(555.51)$ & $3773.31(4441.04)$ & $2571.44(1108.94)$ & 2.240 & 0.152 \\
\hline ED_A & $15.33(6.86)$ & $15.60(7.73)$ & $11.00(5.71)$ & $14.50(4.78)$ & 3.814 & 0.067 \\
\hline ED_T & $2990.18(892.74)$ & $2636.43(610.22)$ & $3496.13(2238.51)$ & $2627.38(1051.16)$ & 0.087 & 0.772 \\
\hline
\end{tabular}

*p<0.05. ADHD: attention-deficit/hyperactivity disorder, ASD: autism spectrum disorder, ED_A: Correct Response for Total Trial, ED_ T: Median Response Time for Total Trials, HAP_CR: Correct Responses for EDF40 Happy Trials, HAPRTCR: Median Response Time for Correct Happy Trials, SAD_CR: Correct Responses for Sad Trials, SADRTCR: Median Response Time for Correct Sad Trials, SD: standard deviation

\section{Comparison between the ADHD and ASD groups for improvement in ability to discriminate facial expression intensity after SST (Table 6)}

The ADHD and ASD groups did not show any significant difference in the ability to discriminate facial expression intensity for either happy or sad expressions. There was also no difference between the two groups for response time, irrespective of the accuracy of response or the type of expression. Neither group showed significant changes in accuracy or response time for discrimination of facial expression intensity after SST.

\section{DISCUSSION}

This study used structured neurocognitive tests to examine changes in facial expression emotion recognition and emotion intensity discrimination after SST in school-age chil- dren with ADHD and ASD, as well as to investigate whether there was any difference between the groups with regards to the extent of improvement.

Following SST, the ADHD group showed positive changes not only in sociability, but also school competence, attention, internalizing problems, and externalizing problems. This is consistent with a previous study that reported that SST improved externalizing and internalizing problems in addition to social skills in children with ADHD [10]. SST has been considered to show limited effects on learning or attention problems in children, since its primary objective is to improve social skills, but some studies have also reported improvements in attention after SST [10]. We also observed improvements in parent-reported attention, which is consistent with both a study done by Paek et al. [9] that demonstrated some improvement in parent-reported attention in addition to sociability, as well as a study by Tutty et al. [29] 
that demonstrated significant improvement in parent-reported attention despite a lack of improvement in teacherreported attention. This improvement in parent-reported attention could be due to training of both parents and children in the SST program, namely with regards to conversation and interaction methods, rule adherence, and task completion; improved performance in daily living; or behavior correction strategies learned by parents during the parent sessions, which may lead to improved performances by children. However, there has still been very little research on cognitive or neuropsychological mechanisms that could explain improvements in attention due to SST, and thus, further studies will be required.

On the other hand, even though the ASD group showed significant changes in the somatic complaints and attention problems subscales from the K-CBCL and in the ARS, they showed no significant changes in scales directly related to sociability, which is the main objective of SST. Although not statistically significant, the ASD group did show a trend-level improvement in the SSRS ( $\mathrm{p}=0.058$ ). This differs slightly from the results of a study by Hwang and Kwak [10], in which, after implementing a SST program similar to that of our study, children with ASD showed improvements in the K-CBCL social competence subscale, as well as in SSRS and MESSY. This discrepancy may be due to a lack of statistical power because of the small number of ASD patients who completed participation in our study, or it may be due to the fact that the children with ADHD in our study showed significantly higher K-CBCL sociability scores than the children with ASD before SST, and in the study by Hwang and Kwak [10], there was no difference between the two groups before SST. Thus, the higher sociability of the children with ASD in that study than those in ours could have affected the results.

Nevertheless, when controlling for the K-WISC-IV Processing Speed Index and K-CBCL social competence subscale, in order to control for the difference between the two groups in sociability before SST, we observed significant differences between the two groups in the change in K-CBCL social problems and MESSY after SST. This means that, even with the same sociability score before SST, there was a significant difference in the extent of improvement in sociability after the program. This could be due to differences in the ability to learn social skills within the program, or in the ability to apply these social skills to daily living. This suggests that in order to improve sociability in children with ASD, it will be necessary to provide longer, more focused, and more detailed SST programs than those provided to children with ADHD, as well as to develop and teach strategies for applying and generalizing the program social skills to daily living.

In our study, the ADHD group showed increased in both
MESSY and SSRS scores, but the ASD group, although not statistically significant, actually showed a decrease in MESSY score and an increase in SSRS score. This may be partly because MESSY contains questions about problem behaviors, whereas in the parent version of the SRSS, which consists of 3 scales (social skills, problem behaviors, academic competence), we only used the 38 questions relating to the social skills scale in our study. This suggests that ADHD group showed a decrease in problem behaviors and an increase in prosocial behaviors, whereas the ASD group showed an increase in prosocial behaviors but did not show a decrease in social problem behaviors. This is consistent with the finding that the social problems subscale in the K-CBCL showed a significant decrease in the ADHD group but did not change in the ASD group. The fact that social problem behaviors did not decrease in the ASD group could be the result of a lack of overall sociability improvement due to the pathophysiology of ASD, for which social deficiency is a core symptom. We also cannot exclude the possibility of error due to the small sample size for the ASD group, which may result in low statistical power. Therefore, it will be necessary to conduct further studies on a larger number of children with ASD and, if similar results are observed, to reinforce contents in order to reduce social problem behaviors in programs for children with ASD.

The SST program in our study did not include any sessions or activities focusing on improving facial expression recognition. Therefore, any improvement in this area would have been learned indirectly while acquiring social skills during the SST period, which would provide evidence for the execution of social skills, rather than direct learning. It is also possible that improvements in facial expression recognition could occur because SST provides the subjects with more interactions with peers and program instructors, which leads to more opportunities to recognize non-verbal cues, including facial expressions. Neither group showed significant improvements in recognizing the type or intensity of facial expressions after the program. However, when the two groups were compared, controlling for the K-WISC-IV Processing Speed Index and K-CBCL social competence subscale, the ADHD group showed a greater increase than the ASD group in the number of correct responses for all expressions combined, female expressions, sad expressions, mild expressions, extreme female expressions, mild male expressions, and Caucasian expressions. This indicates that even if children with ADHD did not directly learn facial expression recognition, their ability to indirectly learn facial expression recognition through social activities was higher than that of the ASD group. Thus, when developing programs with the aim of improving sociability, especially for children with ASD, in 
order to also improve facial expression recognition, indirect methods such as simply increasing opportunities for facial expression recognition must be accompanied by sessions or activities that deal directly with expression recognition skills. Berggren et al. [30] analyzed 13 randomized controlled trials (RCTs) regarding the effects of direct facial expression and emotion recognition training in children and adolescents with ASD and reported that all studies demonstrated significant improvements, supporting the need for sessions or activities dealing directly with facial expression recognition skills. Of the 13 RCTs in this analysis, 4 provided a program to improve other social skills, whereas the other 9 studies only provided a program related to facial expression or emotion recognition. Notably, these 9 studies showed improvements in facial expression recognition but not in parent- or therapist-rated overall social skills, whereas the studies that also included a program to improve social skills reported improvements in both facial expression or emotion recognition and in overall social skills. This demonstrates that, rather than providing only a program that improves facial expression and emotion recognition or only a program that improves general social skills, combining these programs may lead to better outcomes in improved facial expression/emotion recognition and sociability in children with ASD. In the future, it will be necessary to develop programs that include both elements.

When we compared response times for correctly identifying emotions in facial expressions following SST, the ADHD group showed significant reductions in overall response times, as well as in response times to male emotions, neutral emotions, extreme emotions, and extreme male emotions. Conversely, the ASD group showed no changes in response time after SST. Even after controlling for the K-WISC-IV processing speed index and K-CBCL social competence subscale, the two groups showed a significant difference in the improvement in response time to mild female emotions $(\mathrm{p}=0.043)$. This may be because the ADHD group showed a better ability to learn facial expression recognition than did the ASD group and attention significantly improved following the SST program, resulting in faster response times to the same stimuli.

For both happy and sad expressions, we did not observe any differences between the ADHD and ASD groups in the accuracy or response times for discrimination of facial expression intensity. Even after SST, we did not observe any significant changes in either group. This is similar to previous studies, which reported no difference in facial expression intensity discrimination between ADHD and ASD groups, suggesting that this is a more difficult task than facial expression recognition and requires discrimination between more subtle differences. Thus, it appears that children with ADHD find this task as difficult as children with ASD and that there are limitations to improving expression intensity discrimination with SST alone.

Our study had several limitations, including the small number of subjects, the relatively small number of female children compared to male children, the differences in the numbers of children with ASD and ADHD, the fact that there was no control group and the only comparison was between the two disease groups, the fact that we could not include a simple education control group for comparison with SST, and the fact that the reliability and validity of the Penn Emotion Discrimination Task (EDF40) and the Penn Emotion Recognition Task (ER40) have not yet been investigated for Korean children. Although we excluded subjects who attended less than 12 of the 24 SST sessions, due to the small sample size, we could not analyze the effects of child or adult session participation frequency, which could be considered another limitation or factor contributing to this study. Nevertheless, our study possessed many strengths, including the relatively long SST program including a simultaneous program for parents; use of objective neurocognitive tests to measure and compare facial expression recognition and discrimination before and after SST; direct comparison of not only facial expression recognition and discrimination abilities between the two groups, but also the change in these abilities after SST; and extraction of characteristics for each disease group. In this regard, the results of our study are of clinical and academic interest.

\section{CONCLUSION}

In this study, we implemented SST for children with ASD and ADHD and directly compared the ability to recognize emotion in facial expressions and to discriminate expression intensity before and after SST. SST has been shown to be effective at improving sociability, and we observed positive changes in the ADHD group after SST in not only sociability, but also school competence, attention, internalizing problems, and externalizing problems. The ASD group showed a non-significant trend-level improvement in sociability.

Following the SST program, the ADHD group showed greater improvements than the ASD group in overall recognition of facial expressions as well as recognition of sad expressions, mild expressions, extreme female expressions, mild male expressions, and Caucasian expressions. They also showed a greater improvement in response speed. On the other hand, the ASD group showed limited improvement in facial expression recognition, and in particular, the ability to discriminate intensity of facial expressions showed no significant changes 
in either group after SST, with no significant differences between the two groups.

Facial expression recognition plays an important role as a form of reference data for social situations, and in future SST programs, it is important to reinforce audiovisual materials and activities in order to improve this ability. In particular, in programs for individuals with ASD, it is necessary to include even stronger training for facial expression recognition and expression intensity discrimination.

\section{Acknowledgments}

This work was supported by a research grant from a Jeju National University Hospital development fund in 2016.

\section{Conflicts of Interest}

The authors have no financial conflicts of interest.

\section{REFERENCES}

1) Rao PA, Beidel DC, Murray MJ. Social skills interventions for children with Asperger's syndrome or high-functioning autism: a review and recommendations. J Autism Dev Disord 2008;38:353-361.

2) Izard C, Fine S, Schultz D, Mostow A, Ackerman B, Youngstrom E. Emotion knowledge as a predictor of social behavior and academic competence in children at risk. Psychol Sci 2001;12:18-23.

3) Oerlemans AM, van der Meer JM, van Steijn DJ, de Ruiter SW, de Bruijn YG, de Sonneville LM, et al. Recognition of facial emotion and affective prosody in children with ASD (+ADHD) and their unaffected siblings. Eur Child Adolesc Psychiatry 2014;23:257-271.

4) Kuusikko S, Haapsamo H, Jansson-Verkasalo E, Hurtig T, Mattila ML, Ebeling H, et al. Emotion recognition in children and adolescents with autism spectrum disorders. J Autism Dev Disord 2009;39: 938-945.

5) Hobson RP. Methodological issues for experiments on autistic individuals' perception and understanding of emotion. J Child Psychol Psychiatry 1991;32:1135-1158.

6) Downs A, Smith T. Emotional understanding, cooperation, and social behavior in high-functioning children with autism. J Autism Dev Disord 2004;34:625-635.

7) Berggren S, Engström AC, Bölte S. Facial affect recognition in autism, ADHD and typical development. Cogn Neuropsychiatry 2016; 21:213-227.

8) Lee JS, Kang NR, Kim HJ, Kwak YS. Difference of facial emotion recognition and discrimination between children with attention deficit hyperactivity disorder and autism spectrum disorder. J Korean Acad Child Adolesc Psychiatry 2016;27:207-215.

9) Paek MJ, Ahn JK, Lim SY, Kim YR, Park MH, Kim BN, et al. The effectiveness of school based short-term social skills training in children with attention deficit hyperactivity disorder. J Korean Acad Child Adolesc Psychiatry 2009;20:82-89.

10) Hwang JH, Kwak YS. The effect of social skills training for children with attention-deficit hyperactivity disorder and Asperger's disorder: preliminary study. J Korean Acad Child Adolesc Psychiatry 2013;24:199-206.

11) Otero TL, Schatz RB, Merrill AC, Bellini S. Social skills training for youth with autism spectrum disorders: a follow-up. Child Adolesc Psychiatr Clin N Am 2015;24:99-115.

12) Pfiffner LJ, McBurnett K. Social skills training with parent gener- alization: treatment effects for children with attention deficit disorder. J Consult Clin Psychol 1997;65:749-757.

13) Kaufman J, Birmaher B, Brent D, Rao U, Flynn C, Moreci P, et al. Schedule for affective disorders and schizophrenia for school-age children-present and lifetime version (K-SADS-PL): initial reliability and validity data. J Am Acad Child Adolesc Psychiatry 1997;36: 980-988.

14) Kim YS, Cheon KA, Kim BN, Chang SA, Yoo HJ, Kim JW, et al. The reliability and validity of kiddie-schedule for affective disorders and schizophrenia-present and lifetime version-Korean version (K-SADS-PL-K). Yonsei Med J 2004;45:81-89.

15) Koh MK, Noh EA, Kim HW. Korean Wechsler Intelligence Scale for children-fourth edition profiles in child and adolescent with attention-deficit hyperactivity disorder: retrospective study. J Korean Acad Child Adolesc Psychiatry 2015;26:183-189.

16) Oh KJ, Lee HR. Development of Korean child behavior checklist: a preliminary study. J Korean Neuropsychiatr Assoc 1990;29:452-462.

17) DuPaul GJ. Parent and teacher ratings of ADHD symptoms: psychometric properties in a community-based sample. J Clin Child Adolesc Psychol 1991;20:245-253.

18) So YK, Noh JS, Kim YS, Ko SG, Koh YJ. The reliability and validity of Korean parent and teacher ADHD rating scale. J Korean Neuropsychiatr Assoc 2002;41:283-289.

19) Oh KJ, Lee HR. Assessment of ADHD with abbreviated Conners Rating Scale. Korean J Clin Psychol 1989;8:135-142.

20) Shin MS, Cho S, Chun SY, Hong KE. A study of the development and standardization of ADHD diagnostic system. J Korean Acad Child Adolesc Psychiatry 2000;11:91-99.

21) Matson JL, Rotatori AF, Helsel WJ. Development of a rating scale to measure social skills in children: the matson evaluation of social skills with youngsters (MESSY). Behav Res Ther 1983;21:335-340.

22) Park NS, Oh KJ. Effects of methylphenidate treatment on cognitive behavioral symptoms and social, academic \& emotional adjustment of ADHD children. Korean J Clin Psychol 1992;11:235-248.

23) Oh KJ, Min SK, Park JK, Ko RW. Development of cognitive-behavioral treatment for noncompliant and aggressive behavior in children. Korean J Clin Pyschol 1998;17:1-15.

24) Gresham FM, Elliott SN. Social skills rating system: manual. Circle Pines, MN: American Guidance Service;1990.

25) Han ES, Ahn DH, Lee YH. The effects of social skills training in children with attention deficit hyperactivity disorder. J Korean Acad Child Adolesc Psychiatry 2001;12:79-93.

26) Gur RC, Sara R, Hagendoorn M, Marom O, Hughett P, Macy L, et al. A method for obtaining 3-dimensional facial expressions and its standardization for use in neurocognitive studies. J Neurosci Methods 2002;115:137-143.

27) Erwin RJ, Gur RC, Gur RE, Skolnick B, Mawhinney-Hee M, Smailis J. Facial emotion discrimination: I. task construction and behavioral findings in normal subjects. Psychiatry Res 1992;42:231-240.

28) Rojahn J, Gerhards F, Matlock ST, Kroeger TL. Reliability and validity studies of the Facial Discrimination Task for emotion research. Psychiatry Res 2000;95:169-181.

29) Tutty S, Gephart H, Wurzbacher K. Enhancing behavioral and social skill functioning in children newly diagnosed with attentiondeficit hyperactivity disorder in a pediatric setting. J Dev Behav Pediatr 2003;24:51-57.

30) Berggren S, Fletcher-Watson S, Milenkovic N, Marschik PB, Bölte $\mathrm{S}$, Jonsson U. Emotion recognition training in autism spectrum disorder: a systematic review of challenges related to generalizability. Dev Neurorehabil 2018;21:141-154. 